Supplementary Information for:

\title{
Use of NMR-Based Metabolomics To Chemically \\ Characterize the Roasting Process of Chicory Root
}

Feifei Wei $^{\dagger, \ddagger}$, Kazuo Furihata ${ }^{\dagger}$, Mimin Zhang ${ }^{\dagger}$, Takuya Miyakawa ${ }^{\dagger}$ and Masaru Tanokura $^{*} \dagger$

${ }^{\dagger}$ Department of Applied Biological Chemistry, Graduate School of Agricultural and Life Sciences, The University of Tokyo, 1-1-1 Yayoi, Bunkyo-ku, Tokyo 113-8657, Japan

${ }^{\ddagger}$ Japan Society for the Promotion of Science, 8 Ichiban-cho, Chiyoda-ku, Tokyo 102-8472, Japan

Contents:

Figure S1. Chemical structures and NMR correlations of the components of dried raw and roasted chicory root. 
1. 2-methyl-2-propanol

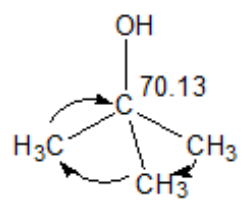

$30.03(1.23)$

3. 5-hydroxymethylfurfural

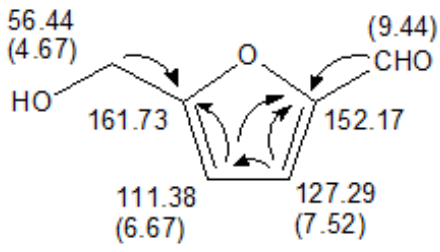

5. acetic acid

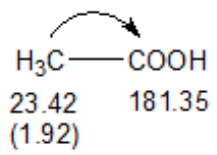

7. arginine

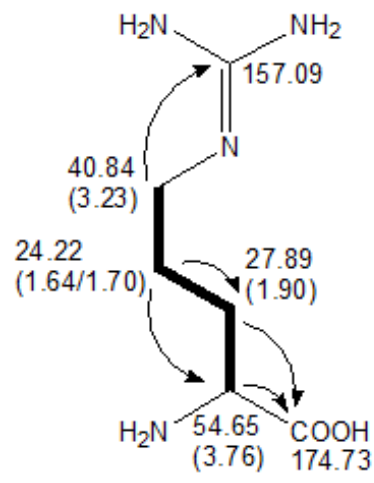

2. 4-amino-N-butyric acid (GABA)

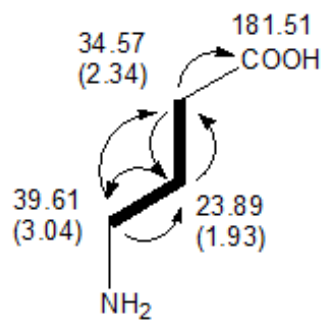

\section{4. acetamide}

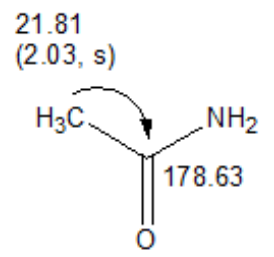

6. alanine

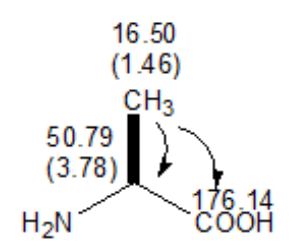

8. asparagine

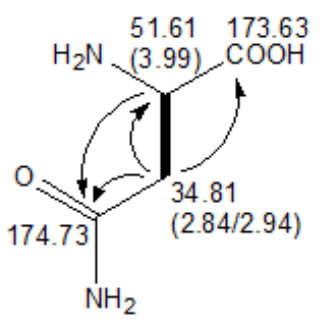

Figure S1. Wei et al.(continued) 


\section{9. chicoric acid}

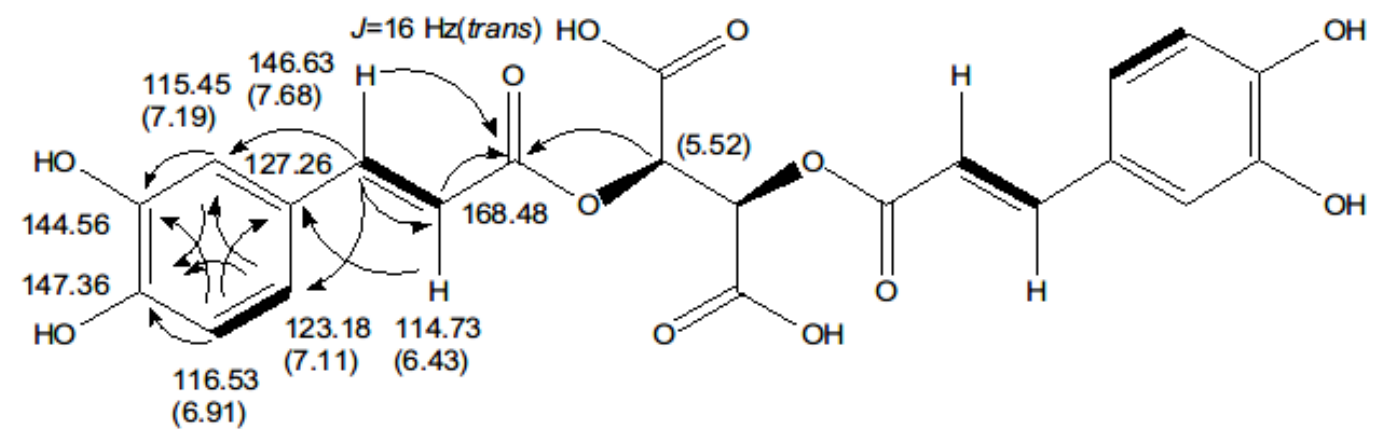

10. choline

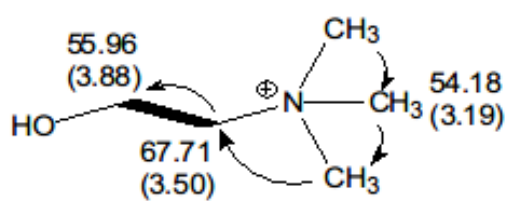

11. citrate

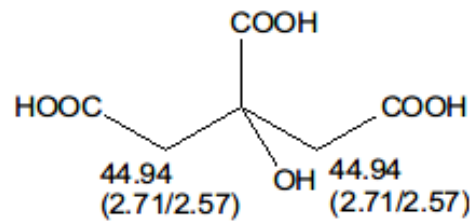

12. di-D-fructose dianhydride: $\alpha$-D-Fruf-1,2':2,1'- $\beta$-D-Fruf

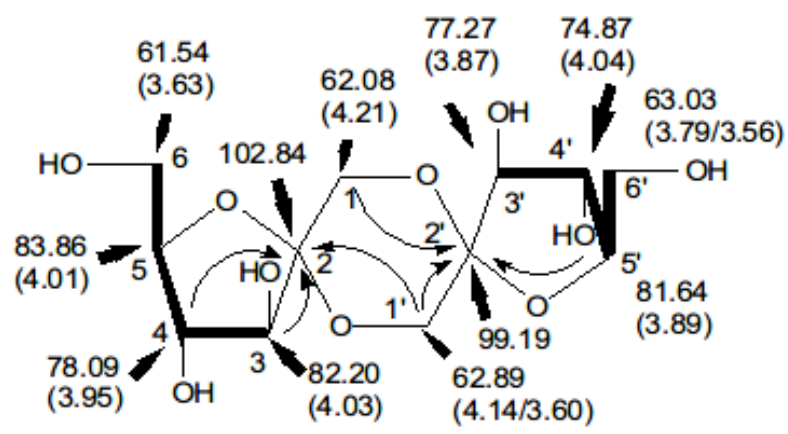

13. ethanol

14. formate

$\mathrm{HCOOH}$

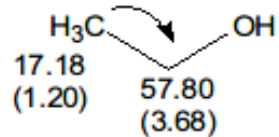

170.67

(8.45)
15. fumarate

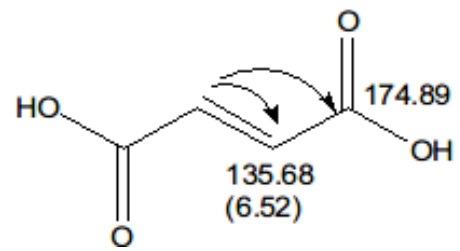

Figure S1. Wei et al. (continued) 


\section{6. glutamine}

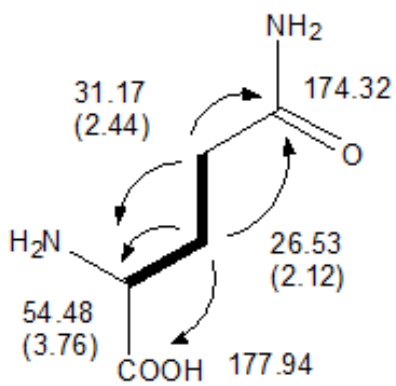

18. inulin-F $m, n$ (chain)

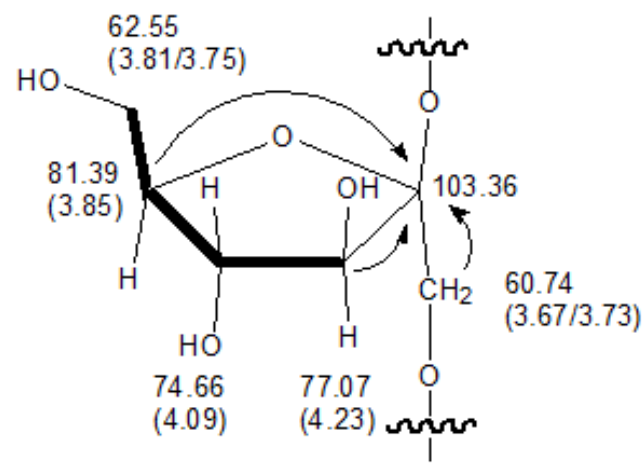

HSQCTOCSY

\section{7. inulin-Fm (terminal)}

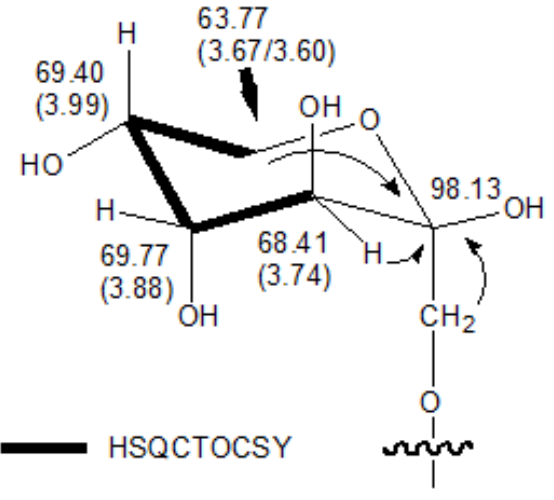

19. inulin-GF $n$ (terminal+1)

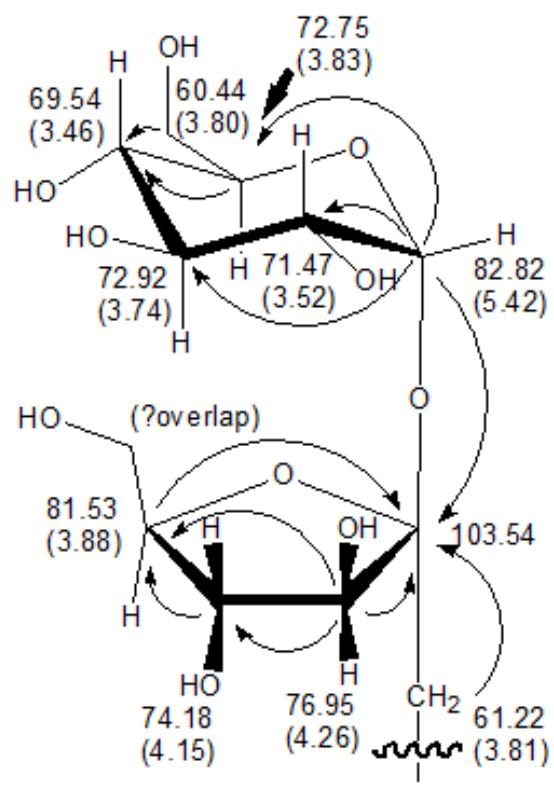

20. isoleucine

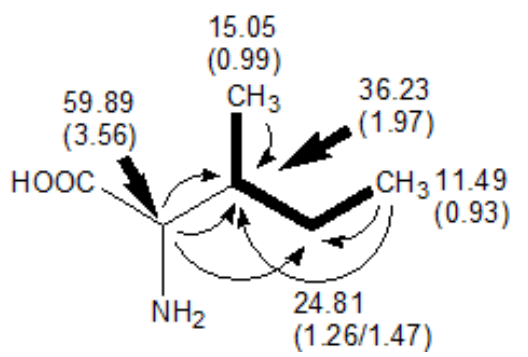

21. lactate
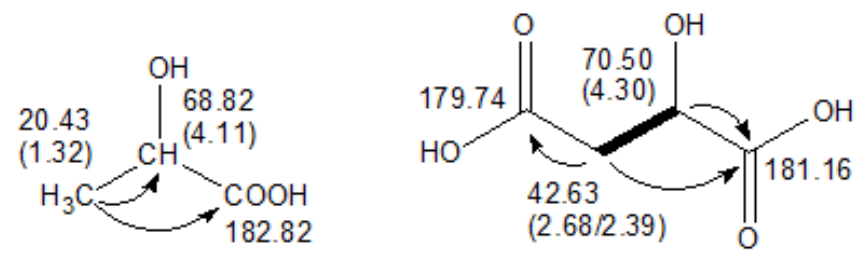

Figure S1. Wei et al. (continued) 
23. methanol

$\mathrm{H}_{3} \mathrm{C}-\mathrm{OH}$
49.26
$(3.34)$

\section{4. monocaffeoyltartarate}

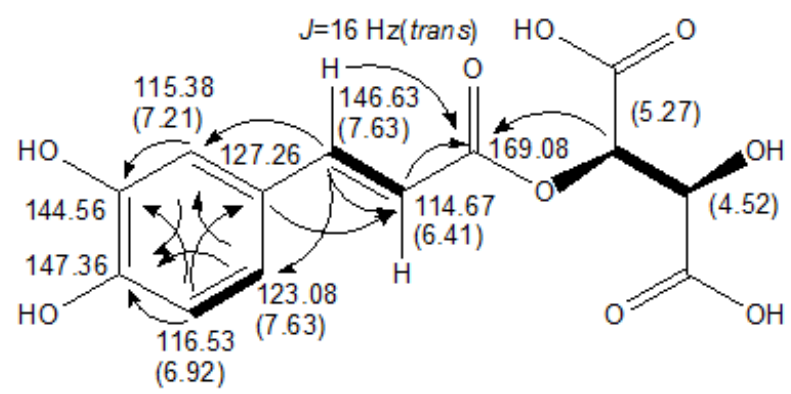

25. 4-hydroxy-5-methyl-2H-furan-3-one 26. phenylalanine

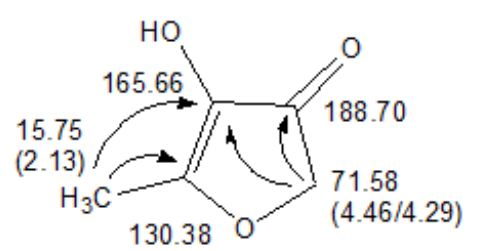

27. proline

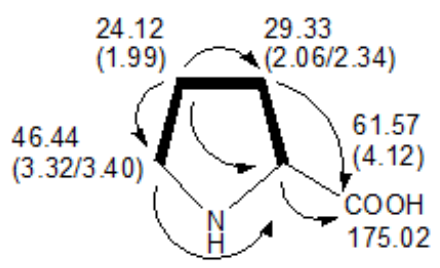

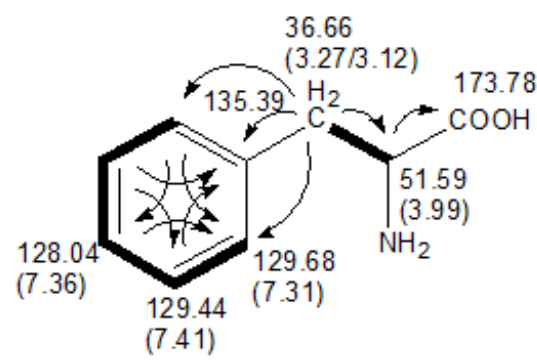

28. pyroglutamate

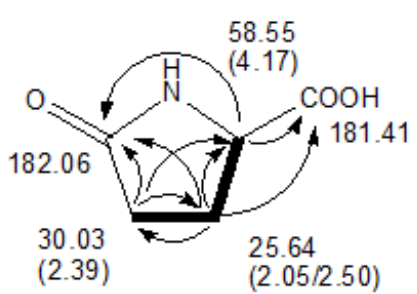

\section{9. sucrose}

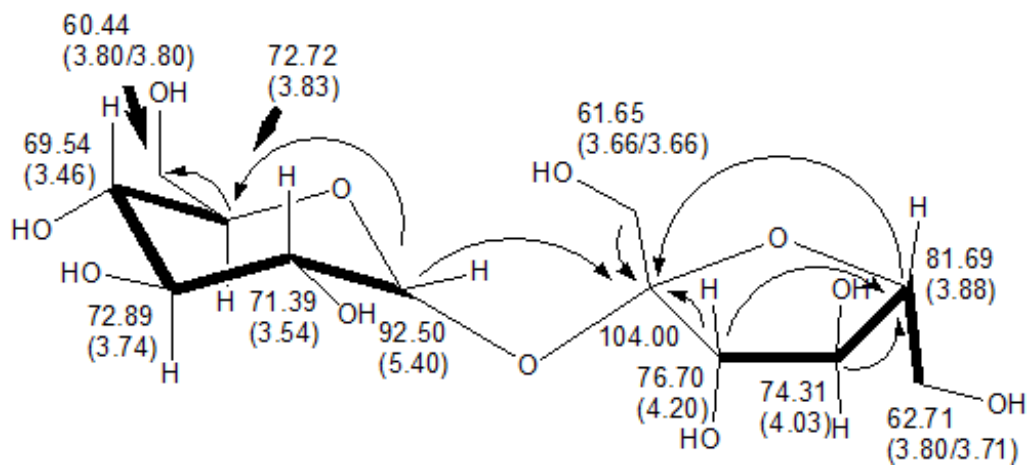

Figure S1. Wei et al. (continued) 
30. threonine

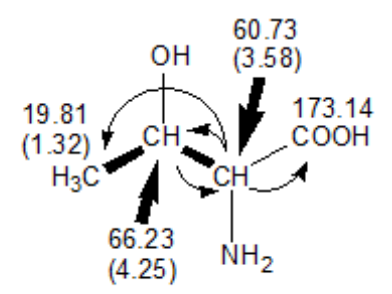

31. trigonelline

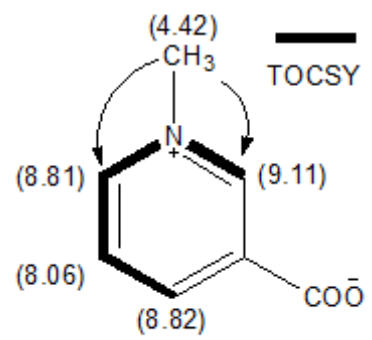

32. valine

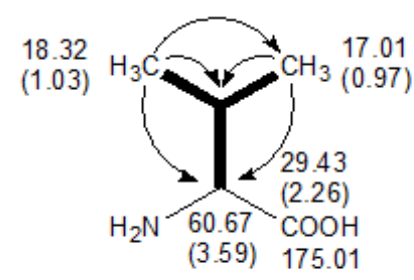

33. $\alpha$-D-glucopyranose

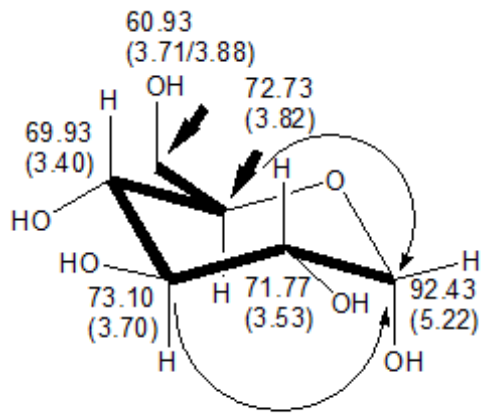

35. $\beta$-D-fructopyranose

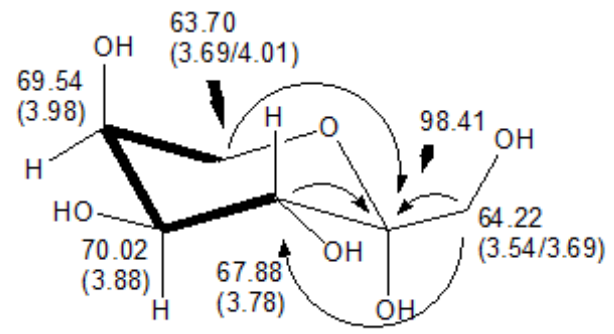

34. $\beta$-D-fructofuranose

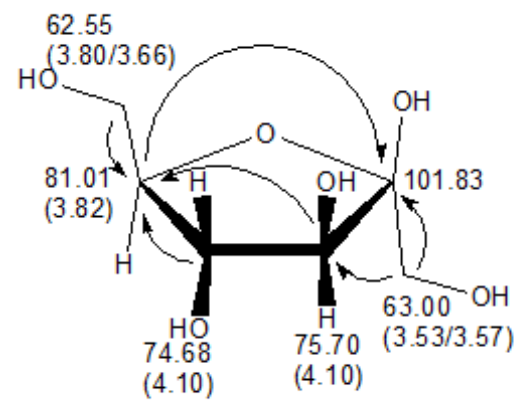

36. $\beta$-D-glucopyranose

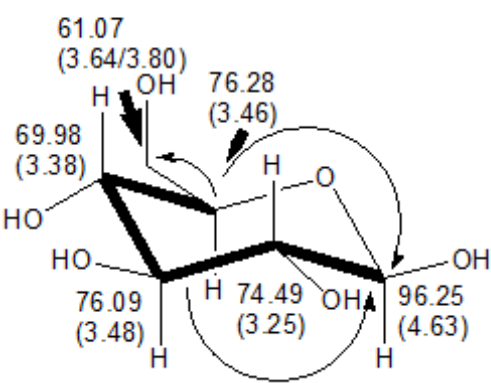

\footnotetext{
- ${ }^{1} \mathrm{H}-{ }^{1} \mathrm{H}$ DQF-COSY; ${ }^{1} \mathrm{H}-{ }^{1} \mathrm{H}$ TOCSY; ${ }^{1} \mathrm{H}-{ }^{13} \mathrm{C}$ HSQCTOCSY $\rightarrow{ }^{1} \mathrm{H}-{ }^{13} \mathrm{C}$ CT-HMBC

$\delta \mathrm{C}(\delta \mathrm{H}) \quad{ }^{1} \mathrm{H}-{ }^{13} \mathrm{C}$ HSQC
}

Figure S1. Wei et al. (continued) 